\title{
Isolated angiitis of the brain in a young female on the contraceptive pill
}

\author{
N. Nagaratnam and W.E. James \\ Department of Medicine, Broken Hill Base Hospital, Broken Hill, N.S.W. 2880, Australia
}

\begin{abstract}
Summary: The case of a 15 year old girl with a rapidly progressing right sided hemiparesis is reported. The history and clinical manifestations were suggestive of either herpes simplex encephalitis or occlusion of the left middle cerebral artery. At necropsy an occlusive thrombosis of the left middle cerebral artery due to a segmental necrotizing granulomatous panarteritis was found. The cause was obscure. A plausible incriminating factor was the contraceptive pill.
\end{abstract}

\section{Introduction}

Vasculitis has always posed problems in diagnosis because of its protean manifestations. In the absence of cutaneous lesions, vasculitis should be considered when there is multiorgan involvement or when there is an unusual clinical presentation.

We would like to report the case of an adolescent girl who presented with a rapidly advancing hemiparesis which was due to a granulomatous panarteritis of the middle cerebral artery. The aetiology of this condition is obscure. However it is noteworthy that our patient had started on the contraceptive pill just 2 weeks prior to her illness.

\section{Case report}

A 15 year old Caucasian female was admitted to hospital with a right sided hemiparesis and diminished conscious state. Twelve hours prior to admission she had developed a generalized headache and felt unwell. A few hours later she was found suffering a generalized tonic-clonic seizure. In hospital she was drowsy, responding to command, had an expressive dysphasia and right hemiparesis. She was normotensive, apyrexial and there was no evidence of meningitis. Laboratory results were as follows: haemoglobin $13.7 \mathrm{~g} / \mathrm{dl}$, packed cell volume $42 \%$, white cell count $10.8 \times 10^{9} / 1$ (neutrophils $55 \%$, lymphocytes $35 \%$ ), platelets $2.05 \times 10^{7} / 1$, erythrocyte sedimentation rate (ESR) $25 \mathrm{~mm} / \mathrm{h}$, prothrombin ratio 1.0 , blood sugar level $6 \mathrm{mmol} / 1$, VDRL negative. Cerebrospinal fluid

Correspondence: W.E. James, M.B., B.S.

Accepted: 10 June 1987
(CSF) protein $0.89 \mathrm{~g} / \mathrm{l}, 30$ neutrophils, no red blood cells, CSF sugar $5 \mathrm{mmol} / \mathrm{l}$.

She was given intravenous dexamethasone but her conscious state continued to deteriorate. A computerized tomogram (CT scan) revealed a left parietal hypodense lesion with mass effect and mid line shift. The next day both pupils suddenly dilated, with only a sluggish response to light from the right pupil. An urgent left parietal burr hole revealed underlying necrotic tissue. Post-operatively there was no evidence of brain stem function and she died 24 hours later, 3 days after the onset of symptoms.

Past medical history revealed a febrile convulsion at 10 months of age, she was a non-smoker and there was no relevant family history. She had suffered no allergies and had been started on Microgynon 30 (levonorgestrel $125 \mu \mathrm{g}$, ethinyloestradiol $50 \mu \mathrm{g}$ ) 2 weeks prior to her illness.

At autopsy there was an area of necrosis in the left temporo-parietal region, sectioning of which revealed an extensive pale infarct in the territory of the left middle cerebral artery. There was a prominent segmental necrotizing vasculitis of the left middle cerebral and right posterior cerebral arteries. An antemortem 5 centimetre clot was flushed from the left internal carotid siphon - this was presumed secondary to distal carotid vasculitic involvement. Lymphocytes, macrophages, epithelioid histiocytes and plasma cells were seen within all three tunics of the affected arterial wall. A few foreign body type giant cells were seen, though well developed granulomata were not revealed. The other intra- and extracranial vessels were normal. Special stains and culture for fungi and acid-fast bacilli were negative. There was no evidence of viral inclusion bodies. Immunofluorescence, microbial and viral cul- 
tures were all unhelpful. Examination of the other organs showed no evidence of vasculitis, infection or other systemic process.

\section{Discussion}

Up to 1986 fewer than 50 well documented cases of isolated angiitis of the central nervous system had been reported. ${ }^{1}$ Vincent ${ }^{2}$ stressed the difficulties in making a correct clinical diagnosis. In an analysis of 26 cases of granulomatous angiitis, he found the majority were in the fifth to eighth decades, and the youngest was two. Hemiparesis and dysphasia were seen as focal cerebral manifestations in 15 patients and seizures occurred during the course of the illness in seven. Cupps et al. ${ }^{3}$ in their study of 18 previously reported cases found the most common presenting symptom was headache. Focal neurological deficits were seen in less than $50 \%$ and seizures in less than $10 \%$ at presentation. In contrast, focal neurological deficits developed in over $90 \%$ of patients during the course of the disease.

Brain biopsy with leptomeningeal sampling is regarded as a useful diagnostic investigation. ${ }^{2}$ Unfortunately brain biopsy in our patient was unrewarding and the leptomeninges were reported as normal macroscopically but were not examined microscopically.

The illness is unpredictable: it can either be an acute fulminating one occurring over a few weeks or it can be more insidious, lasting months. Published experience ${ }^{4}$ is suggestive of an extremely poor prognosis with a fatal outcome in the majority of cases, $90 \%$ at 12 months.

Our patient deteriorated rapidly from the onset and it is interesting that mainly the large and the middle

\section{References}

1. Barnet, J.H.M. (ed) In Stroke: Pathophysiology Diagnosis and Management. Churchill Livingstone, Edinburgh, London 1986, 39: 789-803.

2. Vincent, F.M. Granulomatous angiitis. $N$ Engl J Med 1977, 296: 452.

3. Cupps, T.R., Moor, P.M. \& Fauci, A.S. Isolated angiitis of central nervous system. Prospective, diagnostic and therapeutic experience. Am J Med 1983, 74: 97-105.

4. Sabharwal, U.K., Keogh, L.H., Weisman, M.H. \& Zvaifler, N.J. Granulomatous angiitis of the nervous system: A case report and review of the literature. Arthritis Rheumat 1982, 25: $342-345$. size cerebral vessels were involved. In previous reports no comment has been made on the association of vessel size, onset or severity of the disease. In our opinion small vessel vasculitis is likely to be associated with gradually progressive disease, whilst vasculitis of the medium and large cerebral vessels would be associated with rapidly progressive disease, as in our patient. If this is established it may be a guide as to treatment.

The cause of the angiitis in our patient was obscure. Attention is drawn to the commencement of the oral contraceptive pill 2 weeks prior to the onset of her illness. Drugs such as methamphetamine ${ }^{5}$ and allopurinol can cause vasculitis. ${ }^{6}$ It is well known that oestrogens alter plasma proteins making blood more coagulable. Whether the patient had an incipient vasculitis decompensated by a change in clotting status (precipitated by the pill) or whether the pill itself caused a vasculitis remains to be established.

We have highlighted a number of atypical features in an unusual disease; namely the presentation of the vasculitis as a stroke, ${ }^{7}$ arterial occlusion by thrombus ${ }^{1}$ and segmental granulomatous vasculitis restricted to the large cerebral vessels associated with contraceptive pill intake. These features represent further heterogeneity of isolated angiitis of the central nervous system.

\section{Acknowledgements}

We thank Professor J. Chalmers, Professor of Medicine, Flinders University, Adelaide for permission to use the data, Dr J.M. King, Flinders Medical Centre, for the autopsy findings, Mrs W. Fisk for secretarial work and Dr Dharmavaratha, Clinical Superintendent, Broken Hill Base Hospital, for reading the manuscript.

5. Margolis, M.T. \& Newton, T.A. Methamphetamine ('speed') arteritis. Neuroradiology 1971, 3: 179-182.

6. Weiss, E.R., Forman, P. \& Fosenthal, I.M. Allopurinal induced arteritis in partial H.G.P.R.-tase deficiency. Atypical seizure manifestation. Arch Intern Med 1978, 138: $1743-1744$.

7. Shuangshoti, Sl. Localised granulomatous (giant cell) angiitis of brain with eosinophil infiltration and saccular aneurysm. J Med Assoc Thai 1979, 62: 281. 